\title{
Editorial
}

\section{Progress and Perspectives in Visible-Light-Driven Photocatalysis}

\author{
Monica Baia, ${ }^{1}$ Krisztina Gajda-Schrantz, ${ }^{2}$ Shaohua Shen, ${ }^{3}$ and Elias Stathatos ${ }^{4}$ \\ ${ }^{1}$ Faculty of Physics and Interdisciplinary Research Institute on Bio-Nano-Sciences, Babes-Bolyai University, \\ 400084 Cluj-Napoca, Romania \\ ${ }^{2}$ Department of Inorganic and Analytical Chemistry, Environmental Chemistry Research Group, University of Szeged, \\ Dóm tér 7, Szeged 6720, Hungary \\ ${ }^{3}$ International Research Center for Renewable Energy, State Key Laboratory of Multiphase Flow in Power Engineering, \\ Xian Jiaotong University, Shaanxi 710049, China \\ ${ }^{4}$ Electrical Engineering Department, Technological-Educational Institute of Western Greece, 26334 Patras, Greece
}

Correspondence should be addressed to Monica Baia; monica.baia@phys.ubbcluj.ro

Received 23 October 2013; Accepted 23 October 2013

Copyright (C) 2013 Monica Baia et al. This is an open access article distributed under the Creative Commons Attribution License, which permits unrestricted use, distribution, and reproduction in any medium, provided the original work is properly cited.

The development of nanotechnology for the synthesis of nanomaterials is providing unprecedented opportunities to deal with emerging environmental problems associated with water and air contamination along with worldwide energyrelated concerns. Advanced oxidation technologies (AOTs) and nanotechnologies (AONs) have been extensively investigated for the destruction of toxic and recalcitrant organic compounds and inactivation of microorganisms in water and air. Photocatalysis as a part of AOTs is an effective method to completely decompose organic pollutants in air and aqueous solutions/natural waters. However, conventional wide band gap semiconducting materials $\left(\mathrm{TiO}_{2}, \mathrm{ZnO}\right.$, etc.) usually employed in photocatalytic processes absorb radiation below $400 \mathrm{~nm}$, which is in the UV region, being only $5 \%$ of the solar light. In order to effectively utilize solar light as the source of energy, modified materials that can also absorb in the visible spectrum need to be synthesized. Recently, doping $\mathrm{TiO}_{2}$ with different heteroatoms (metal and/or nonmetal ions) made it possible to shift the absorption towards longer wavelengths and, thus, allow $\mathrm{TiO}_{2}$ sensitization in the visible region. Due to the visible light absorption abilities, doped $\mathrm{TiO}_{2}$-based powders and films can also be used for improving the photocatalytic process in the visible region. Several attempts have been directed towards the development of modified $\mathrm{TiO}_{2}$ with visible light response by dye sensitization, metal (Fe, Co, Ag), and nonmetal (N, F, $\mathrm{C}, \mathrm{S}$ ) doping of the catalyst to reduce $\mathrm{TiO}_{2}$ band gab energy requirements for photocatalytic activation. In some metal doping approaches, the resulting visible light photocatalytic activity has some drawbacks including increase in the carrierrecombination centers (electron-hole pair species generated after photoexcitation of the catalyst) and low thermal stability of the modified material. Furthermore, the sensitization of $\mathrm{TiO}_{2}$ nanoparticles with quantum dots of CdS, CdSe, CdTe, $\mathrm{PbS}$, and $\mathrm{PbSe}$ and their combinations could effectively lead to visible-light-activated photocatalysts. Several intrinsic visible-light-active semiconductors, mostly metal sulphides, have been fabricated by nonmetal-mediated band structure engineering. The visible-light sensitisation of metal sulphides can be attributed to the $S 3 p$ states, which increase the width of the valence band itself and cause the decrease in the band gap energy. Among the available sulphides, CdS with band gap of $2.4 \mathrm{eV}$ is probably the best-studied metal sulphide photocatalyst, although it is unstable and toxic. The aim of this special issue is to present some recent progress in the field of visible-light-activated photocatalysts. The issue contains ten selected papers after invitation. A brief summary of all accepted papers is provided below.

The article entitled "Preparation of Fe-doped $\mathrm{TiO}_{2}$ nanotubes and their photocatalytic activities under visible light" reported the preparation of $\mathrm{Fe}$-doped $\mathrm{TiO}_{2}$ nanotubes (Fe-TNTs) by ultrasonic-assisted hydrothermal method. The structure and composition of the as-prepared $\mathrm{TiO}_{2}$ nanotubes were characterized by transmission electron 
microscopy (TEM), X-ray diffraction (XRD), and UV-Vis absorption spectroscopy. Their photocatalytic activities were evaluated by the degradation of methyl orange under visible light. The Fe-TNTs demonstrated good photocatalytic activities and photostability under visible light irradiation, and the optimum molar ratio of Ti: Fe was found to be $100: 1$.

In the paper entitled "Facile preparation of phosphotungstic acid-impregnated yeast hybrid microspheres and their photocatalytic performance for decolorization of azo $d y e$ " phosphotungstic acid (HPW)-impregnated yeast hybrid microspheres were prepared by impregnation-adsorption technique through tuning $\mathrm{pH}$ of the aqueous yeast suspensions. The obtained products were characterized by field emission scanning electron microscopy (FE-SEM), energy dispersive spectrometry (EDS), XRD, thermogravimetrydifferential scanning calorimetry (TG-DSC), and UV-Vis spectrophotometry. FE-SEM and EDS ascertained that the HPW has been effectively introduced onto the surface of yeast, and the resulting samples retained ellipsoid shape, with the uniform size (length $4.5 \pm 0.2 \mu \mathrm{m}$, width $3.0 \pm 0.3 \mu \mathrm{m}$ ) and good monodispersion. XRD pattern indicated that the main crystal structure of as-synthesized HPW@yeast microsphere is Keggin structure. TG-DTA stated that the HPW in composites has better thermal stability than pure HPW. Fourier transform infrared spectroscopy (FT-IR) elucidated that the functional groups or chemical bonds inherited from the pristine yeast cell were critical to the assembling of the composites. UV-Vis spectra analysis showed that the obtained samples have a good respondse to UV light. The settling ability indicated that the hybrid microspheres possess an excellent suspension performance. It was shown that the HPW@yeast microsphere exhibited a high photocatalytic activity for the decoloration of Methylene blue and Congo red dye aqueous solutions.

The paper entitled " $\mathrm{Au}-\mathrm{TiO}_{2}$ nanocomposites and efficient photocatalytic hydrogen production under UV-Vis and visible light illuminations: a comparison of different crystalline forms of $\mathrm{TiO}_{2}$ " reported the preparation of Au-loaded $\mathrm{TiO}_{2}$ nanocomposites by the solvated metal atom dispersion (SMAD) method. The obtained samples were characterized by diffuse reflectance UV-vis spectroscopy, powder XRD, BET surface analysis measurements, and TEM bright field imaging. The particle size of the embedded Au nanoparticles ranged from 1 to $10 \mathrm{~nm}$. The nanocomposites were used for photocatalytic hydrogen production in the presence of a sacrificial electron donor like ethanol or methanol under UVvis and visible light illumination. They showed very good photocatalytic activity toward hydrogen production under UV-vis conditions, whereas under visible light illumination, there was considerably less hydrogen produced. Au/P25 gave a hydrogen evolution rate of $1600 \mu \mathrm{mol} / \mathrm{h}$ in the presence of ethanol ( $5 \mathrm{vol} \%)$ under UV-vis illumination. In the case of $\mathrm{Au} / \mathrm{TiO}_{2}$ nanocomposites, the presence of $\mathrm{Au}$ nanoparticles serves two purposes: being an electron sink, to gather electrons from the conduction band of $\mathrm{TiO}_{2}$ and being a reactive site for water/ethanol reduction to generate hydrogen gas. Hydrogen production by water splitting in the absence of a sacrificial electron donor using $\mathrm{Au} / \mathrm{TiO}_{2}$ nanocomposites under UV-vis illumination was also observed.
The paper entitled "Bulky macroporous $\mathrm{TiO}_{2}$ photocatalyst with cellular structure via facile wood-template method" reported the preparation of bulky macroporous $\mathrm{TiO}_{2}$ particles with cellular structure in the presence of wood slices as template. The prepared samples were characterized by SEM, XRD, EDS, and TEM techniques. XRD pattern confirmed that the crystalline phase of the wood-templated $\mathrm{TiO}_{2}$ is anatase phase. SEM image analysis revealed that the wood-templated $\mathrm{TiO}_{2}$ inherited the initial cellular structures of birch lumber (B. albosinensis Burk), and numerous macropores were observed in the sample. Meanwhile, the wood-templated $\mathrm{TiO}_{2}$ presented a superior photocatalytic ability to decompose Rhodamine $\mathrm{B}(\mathrm{RhB})$ under ultraviolet irradiation.

In the paper entitled "Preparation of nanostructured $\mathrm{Cu}_{2} \mathrm{SnS}_{3}$ photocatalysts by solvothermal method" nanostructured $\mathrm{Cu}-\mathrm{Sn}-\mathrm{S}$ powder was prepared by a relatively lowcost, simple, and green solvothermal method. Flower-like $\mathrm{Cu}_{2} \mathrm{SnS}_{3}$ nanostructures were successfully synthesized in $50 \mathrm{vol} \%$ ethanol water solution at $200^{\circ} \mathrm{C}$ for $7.5 \mathrm{~h}$. The structure and photophysical properties of the as-obtained samples were characterized by SEM, TEM, XRD, and UVVis diffusion reflectance spectroscopy. Results showed that the cubic and tetragonal $\mathrm{Cu}_{2} \mathrm{SnS}_{3}$ was obtained by varying the ethanol contents. The band-gap energy of tetragonal $\mathrm{Cu}_{2} \mathrm{SnS}_{3}$ nanocrystals is near the optimum for photovoltaic solar conversion in a single band-gap device.

In the paper entitled "In situ measurement of local hydrogen production rate by bubble-evolved recording" optical microscopy of superfield depth was used for recording the hydrogen bubble growth on $\mathrm{Cd} 0.5 \mathrm{Zn} 0.5 \mathrm{~S}$ photocatalyst in reaction liquid and illuminated with purple light. By analyzing the change of hydrogen bubble size as a function of time, it was revealed that hydrogen bubble growth experienced two periods. The tendency of hydrogen bubble growth was similar to that of the gas bubble in boiling, while the difference in bubble diameter and growth time magnitude was greater. Meanwhile, the local hydrogen production rate on photocatalyst active site was established by measuring hydrogen bubble growth variation characteristics. This method allowed confirming local actual hydrogen evolution rate quantitatively during photocatalytic water splitting.

In the paper entitled "Facile growth of porous hematite films for photoelectrochemical water splitting" a simple fabrication method of porous hematite films with tunable thickness in an aqueous solution containing $\mathrm{FeCl}_{3}$ as the single precursor was introduced. It was demonstrated that the optimized thickness was necessary for high performance photoelectrochemical water splitting, by balancing photon absorption and charge carrier transport. The highest photocurrent of ca. $0.15 \mathrm{~mA} \mathrm{~cm}^{-2}$ at $1.0 \mathrm{~V}$ versus $\mathrm{Ag} / \mathrm{AgCl}$ was achieved on the $300 \mathrm{~nm}$ thick porous hematite film as photoanode, with IPCE at $370 \mathrm{~nm}$ and $0.65 \mathrm{~V}$ versus $\mathrm{Ag} / \mathrm{AgCl}$ to be $9.0 \%$. This simple method allowed the facile fabrication of hematite films with porous nanostructure for enabling high photon harvesting efficiency and maximized interfacial charge transfer. These porous hematite films could be easily modified by metal 
doping for further enhanced photoelectrochemical activity for water splitting.

In the paper entitled "Structural and photoelectrochemical properties of $\mathrm{Cu}$-doped $\mathrm{CdS}$ thin films prepared by ultrasonic spray pyrolysis" $\mathrm{Cu}$-doped $\mathrm{CdS}$ thin films of variable doping levels were deposited on indium tin oxide-coated glass substrate by simple and cost-effective ultrasonic spray pyrolysis. The influences of doping concentration and annealing treatment on the structure and photoelectrochemical properties of the films were investigated. The deposited films were characterized by XRD, SEM, and UV-Vis spectra, and were further investigated by electrochemical and photoelectrochemical measurements with regards to splitting water for solar energy conversion. The results showed that the $\mathrm{Cu}$ impurity can cause a structural change and red shift of the absorption edge. It was found that the photocurrent can be improved by the $\mathrm{Cu}$-doping process for the unannealed films under the weak illumination. The unannealed 5 at.\% $\mathrm{Cu}$-doped sample obtained the maximum IPCE, which achieved about $45 \%$ at $0.3 \mathrm{~V}$ versus SCE potential under $420 \mathrm{~nm}$ wavelength photoirradiation. In addition, the p-type CdS was formed with a doping of 4 at. $\% \sim 10$ at. $\% \mathrm{Cu}$ after $450^{\circ} \mathrm{C} 2 \mathrm{~h}$ annealed in vacuum.

In the paper entitled " $\mathrm{TiO}_{2}$ and $\mathrm{ZnO}$ nanoparticles in photocatalytic and hygienic coatings" antimicrobial paints based on the aqueous acrylic dispersion and various nanoparticles of zinc oxide and titanium dioxide were obtained. Antimicrobial ability and photoactivity were assumed in these paints. It was found that the coating containing the mixture of the first type of $\mathrm{TiO}_{2}$ and nano- $\mathrm{ZnO}$ showed the best photocatalytic effect despite the fact that the first type of $\mathrm{TiO}_{2}$ was not better in the photocatalytic test than the other types of $\mathrm{TiO}_{2}$. The agar dilution method was used to test antimicrobial ability. Escherichia coli, Pseudomonas aeruginosa, and Staphylococcus aureus were chosen as test bacteria and Penicillium chrysogenum and Aspergillus niger as test molds. The antimicrobial effect of coatings with the mixture of the first type of $\mathrm{TiO}_{2}$ and nano- $\mathrm{ZnO}$ was the best of all the tested samples.

The paper entitled "Effect of PdS on photocatalytic hydrogen evolution of nanostructured CdS under visible light irradiation" reported the preparation of nanostructured PdS/CdS by an in situ coprecipitation and hydrothermal method, respectively, in order to investigate the effect of PdS as a cocatalyst for photocatalytic hydrogen evolution. The asprepared photocatalysts were characterized by TEM, XRD, UV-vis absorption spectroscopy, and photoluminescence. With PdS highly dispersed in CdS nanostructures, the photoactivity was evaluated by hydrogen evolution from aqueous solution containing $\mathrm{Na}_{2} \mathrm{~S} / \mathrm{Na}_{2} \mathrm{SO}_{3}$ as sacrificial reagents under visible light irradiation. When the concentration of PdS was $1 \%$ by weight, $\mathrm{PdS} / \mathrm{CdS}$, prepared by the in situ coprecipitation, showed the highest photocatalytic activity, while that prepared by hydrothermal method showed the highest stability for hydrogen evolution. The effect of highly dispersed PdS on the photoactivity was discussed.

\section{Acknowledgments}

We would like to express our thanks to all authors who made this special issue possible. We hope the readers of this issue will find interesting information on the materials and visible light activated photocatalytic processes.

Monica Baia Krisztina Gajda-Schrantz Shaohua Shen Elias Stathatos 

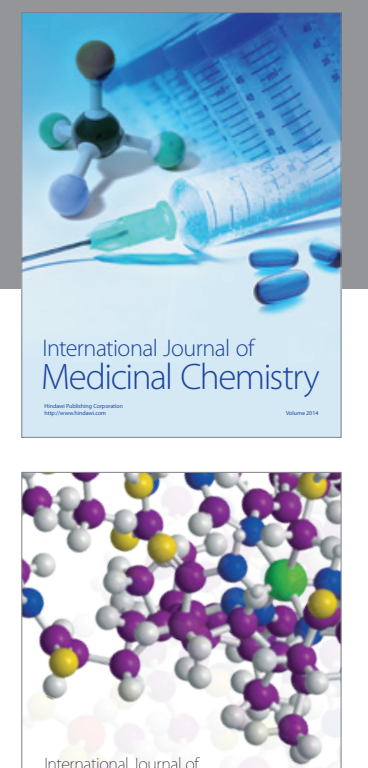

\section{Carbohydrate} Chemistry

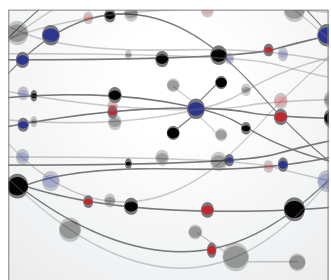

The Scientific World Journal
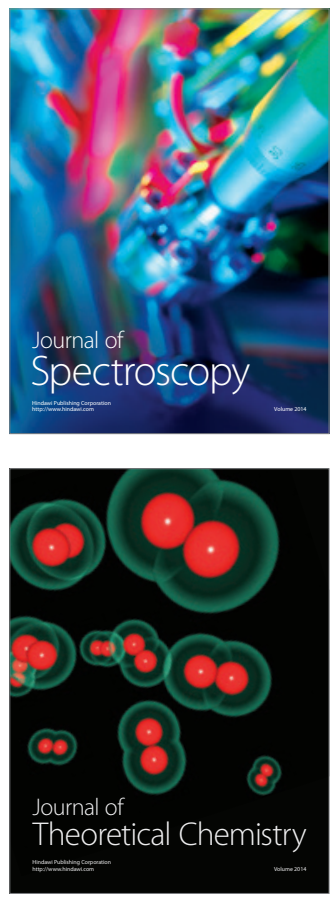
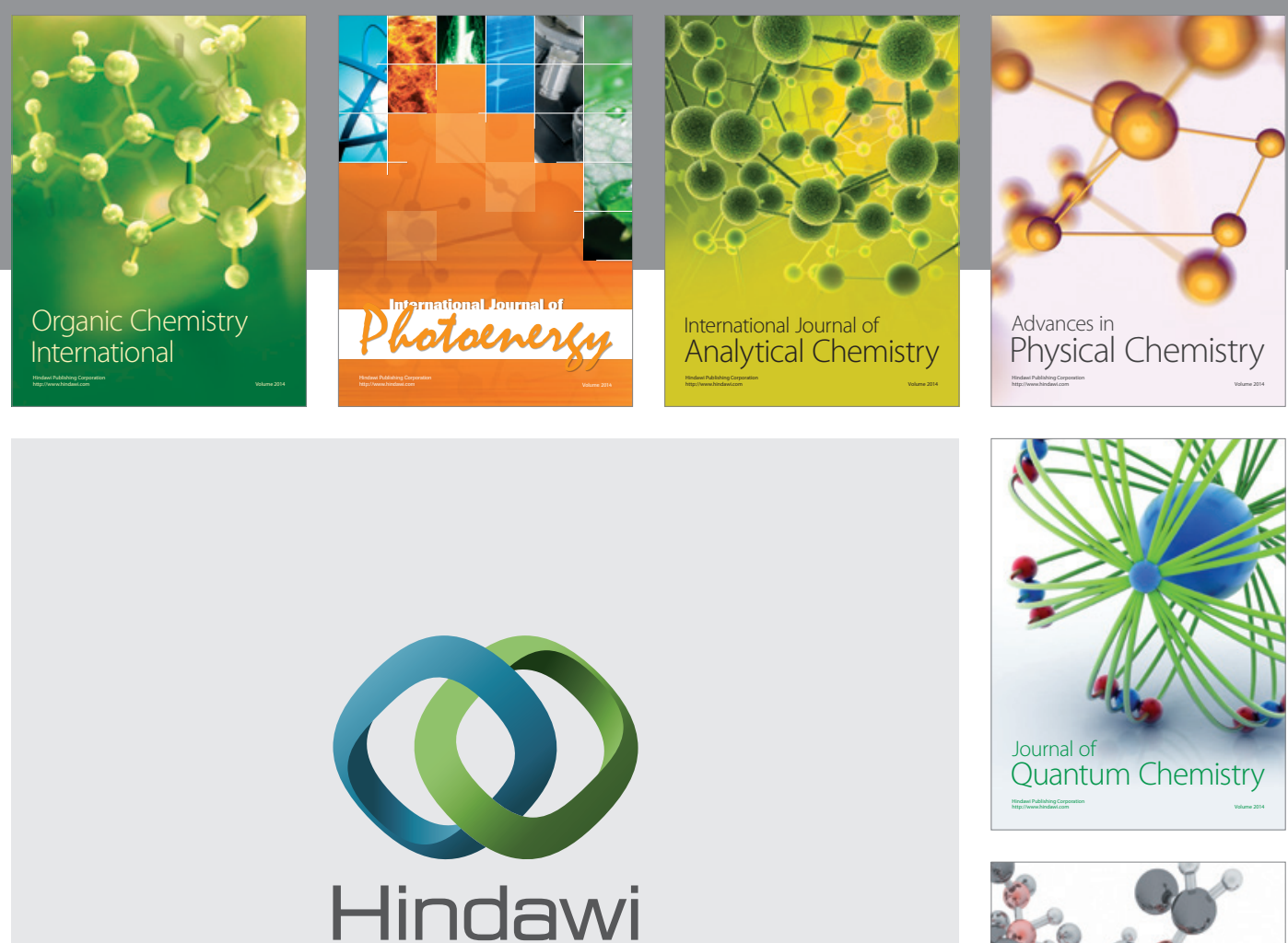

Submit your manuscripts at

http://www.hindawi.com

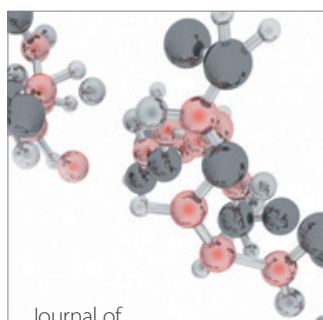

Analytical Methods

in Chemistry

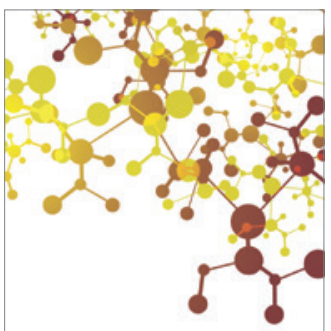

Journal of

Applied Chemistry

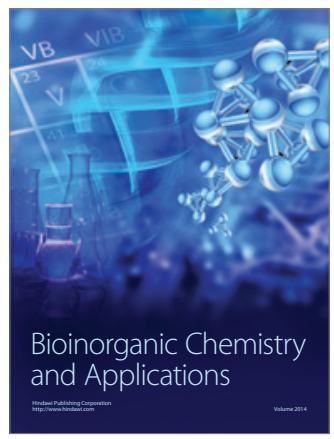

Inorganic Chemistry
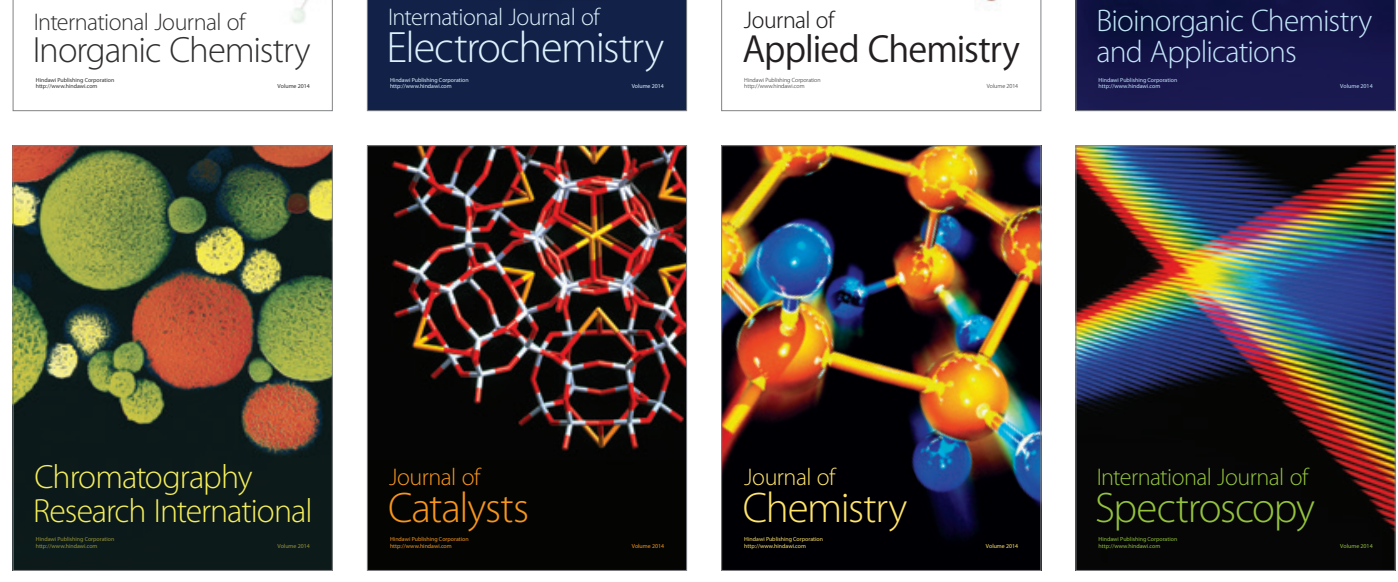